\title{
Autonomous Mobile Robots in High Occupancy Aerospace Manufacturing
}

\author{
Lancelot Martin ${ }^{\mathrm{a}, 1}$, James $\mathrm{Gao}^{\mathrm{a}}$, Alister Wilson ${ }^{\mathrm{b}}$, Chi Hieu Le ${ }^{\mathrm{a}}$, Marcus de Ree \\ ${ }^{a}$ University of Greenwich, Central Ave, Gillingham, Chatham ME4 4TB, UK \\ ${ }^{\mathrm{b}}$ BAE Systems, Marconi Way, Chatham, Rochester ME1 2XX, UK \\ ${ }^{\mathrm{c}}$ IT - Instituto de Telecomunicações, 3810-193 Aveiro, Portugal
}

\begin{abstract}
Using a combination of active Radio-Frequency Identification tracking and staff interviews with members from an aerospace manufacturing company, it was uncovered that over 80 hours per week was spent in the manual movement of goods between departments. On a site of over 1000 employees that uses dedicated build cells in separated departments, this mixed-use facility proves challenging for the adoption of an autonomous delivery system due to its narrow corridors and high occupancy, however by investigating the concerns of employees and suggesting low-cost retroactive solutions, this project seeks to justify the transition from manual to automated onsite logistics. The conclusion found that indeed the company does have the transport yields to justify the use of Autonomous Mobile Robots, that the robots would supplement rather than replace workers and that safety was a key factor to address when using robots on a site of this configuration.
\end{abstract}

Keywords. Autonomous Mobile Robot, Automatic Guided Vehicle, Active RadioFrequency Identification, Onsite logistics, Global navigation, Industry 4.0, Internet of Things

\section{Introduction}

This research was carried out in collaboration with BAE Systems Electronic Systems (Rochester, UK), which is a tier 1 defence and aerospace engineer-to-order contractor. As a high value low-volume manufacturer, the company is constantly looking to improve their production facility, this time with the use of Industry 4.0 technologies.

The main research issues were raised by members of the operations team. BAE Systems had previously investigated a specific technology, Automatic Guided Vehicles (AGVs) circa 2016, however, due to large ramps and safety concerns, the project was scrapped. Now with the technological transition from mobile AGVs to Autonomous Mobile Robots (AMRs), vast improvements in safety had been made because of superior onboard sensors as well as intelligent route planning compared to the static routes of AGVs. When the idea was raised again in 2019 as part of this study, similar concerns were raised, specifically regarding the volume of movement on site, and the safety of a system when implemented site wide rather than to a specific department.

Three methods were implemented when considering the justification of an onsite AMR logistics network. The first was a study of the onsite logistics network, counting the journeys required to transport material between process areas in the building and

\footnotetext{
${ }^{1}$ Corresponding Author.1m9602p@gre.ac.uk
} 
determining the amount of time spent on transport. Then it was the staff reaction to mobile robots (whilst considering their existing knowledge), how these reactions change during exposure to robots and how best to address their concerns. Finally, all safety issues around the use of the robot on site had to be addressed, maintaining the current levels of safety present in the existing manual system.

\section{Literature Review}

The questions manufacturing companies seek to answer when undertaking a project such as this relate to: the functionality of the AMR and the safety of the robot. In regards to functionality, onboard sensors and software are already at a point that collaborative mobile robots can be integrated to a company for many different jobs [1]. In a more nuanced sense that deals with the practicality of having robots on site, robot manufacturing standards dictate that AGVs and AMRs are to be given priority when moving within a manufacturing facility [2]. As such the shortest available route tends to be the deciding factor when planning the journey of a mobile robot through the fleet management system [3]. Where facilities have very high occupancy numbers due to mixed use or a reliance on manual operators, these occupants can impede a robot's journey by triggering emergency stop sensors that prevent collisions [4]. In the case of AGVs, designers will intentionally cut off routes that are inefficient [5] [6]. This could be due to tight corridors or areas of high foot traffic, however, since the introduction of AMRs, route planning has become significantly more dynamic as they are not confined to a track [7]. This freedom to travel down corridors and through confined spaces comes with limitations as occupants are still capable of stopping the robot on route. Priority will fall in favour of wheelchair users in tight spaces as per building regulations and general right of way in these areas is widely seen in favour of the human occupant. Consideration of fleet size relative to humans and cost have also been investigated and shown to be precalculatable using simulation [8] [9].

In the context of delivery robots, robots are a clear example of how technological developments give rise to legislative gaps [10]. There are no explicit laws around autonomous robots, other than the standards set and promised by manufacturers. In a report issued by the House of Lords [11] explained that "while AI-specific regulation is not appropriate at this stage... Existing regulators are best placed to regulate AI in their respective sectors." It can also be seen in the USA, that companies that are at the forefront of these issues often help to shape the future of the legislation around them such as Estonian AMR company Starship [10]. Supplementary standards and regulations also appear in the form of British Standards (BS), International standards Organization (ISO) documents and Health and Safety Executive (HSE) documents [12][13][14][15]. Although these are not explicitly laws, they can be cited in court to determine whether a company was diligent in ensuring the safety of its employees in the event of an accident.

\section{Industrial Investigation and Proposed Methodology}

A combination of qualitative and quantitative data was gathered to justify the use of AMRs onsite. These methods involved collecting tracking data for the existing trolley movements on site to determine exactly how much time engineers were spending to take parts, sub-assemblies, and assemblies to and from different departments. The next was 
to investigate data provided by BAE Systems in the form questionnaire results and focus group discussions. Finally using a literature review of current BS's, HSE and ISO documents, a framework for the AMR's safety level was taken into consideration.

\subsection{Active RFID trolley tracking}

BAE Systems employs a commercial asset tracking system site wide for the tracking of over 2000 assets at any given time. These assets are tracked using asset tags that are affixed to an object which continually emit an active Radio-Frequency Identification (RFID) ping. This ping is registered by an asset tracking hub and the relative distance is calculated based on the time the signal takes to be sent by multiple hubs to the tag and then be sent back with authentication (accounting for building fabric, heights, etc.). It was coincidental that BAE Systems tagged several of its trolleys, providing in some cases years of existing trolley data.

A total of 6375 data points from 39 trolleys between $14^{\text {th }}$ and the $21^{\text {st }}$ of February 2020 (Pre-COVID-19 lockdown data) were analysed. A journey was defined as:

$$
\begin{gathered}
\sum_{\left(P_{x+1}-P_{x}\right) \leq 15 \text { min }}^{J_{\text {time }}}=\text { Journey time }(H H: M M: S S) \\
\sum_{\left(P_{2}-P_{1}\right) \leq 15 \text { min }} J_{\text {time }}=\left(P_{2}-P_{1}\right)+\left(P_{3}-P_{2}\right)+\cdots+\left(P_{x+1}-P_{x}\right) \\
\text { Where } P_{1}=\text { Time at Position one }(H H: M M: S S) \\
\text { Where } P_{2 \rightarrow x}=\text { Time at next Position }(H H: M M: S S)
\end{gathered}
$$

This definition was turned into a visual basic macro with the assistance of visiting academic Dr Marcus de Ree. Within the 354 journeys 54 repetitions were found. Of these 54 repetitions (defined as routes) each route was given a number and an investigation into that department then took place. The data also showed that all movement occurred between 06:00 and 21:00 despite BAE Systems manufacturing on a 24 hours work cycle.

The average daily journey time was 8 minutes, 54 seconds, with an additional 5 minutes being allowed for registering the items handover and the time taken for a staff member to return to their workstation. 354 journeys per week at approximately 15 minutes per journey gives a weekly transport time of 88.5 hours. It was also discovered that several more trolleys (up to a total of 60) had not been tagged for data harvesting, leading to speculation that the amount of time spent on movement may be heavily undervalued.

\subsection{Questionnaire and Focus Group study}

A focus group was carried out by BAE Systems staff members to determine how people would react to the technology. As part of the company culture and the desire to take regular feedback from staff, BAE Systems has set up an existing pool of contributing members known as the Rochester Involvement Group. This is an open group that is available for all full-time staff to join.

Eight engineers all working in the area AMRs were suggested to be implemented and of more than 2 years site experience participated in both the questionnaire and focus group. A three-part questionnaire was sent to the group prior to them meeting. Part-one reviewed the groups knowledge on the current trolley system, their experience with it 
and their general conduct when moving between departments on site. Part-two asked about any existing knowledge participants may have on robots, not specifically AMRs. Finally, Part-three asked specifically about robots on the Rochester site. Overall results showed consistency in the group with a clear understanding of the onsite rules and norms, the ability to differentiate between collaborative and non-collaborative robots and a feeling of safety at the idea of working with robots. Only half of the participants had ever seen a robot in person and half believed there was any real benefit to robots being used at BAE Systems.

The participants then met and were shown two videos during an online session where they could fill out an online poll and openly share opinions and discuss what they saw. The questionnaire showed that all participants felt safe working with robots on site, and this feeling was unchanged after watching the informative videos. Some notable comments from the group after being shown the videos:

- "We don't operate at a speed or volume that could benefit Rochester."

- "(Sites shown in the video are) Not Comparable because of high levels of space. Corridors are not necessarily straight. It would be hard for members to step aside. Issues of right of way. Security doors, lifts, stairs and ramps."

- "The primary concerns are end to end (loading and unloading). Journeys themselves are expected to be completed without problems."

The overall experience with the group was useful as it confirmed many of the suspicions upper management had felt during the interview stages. Each of the comments were refutable, the issue of volume had been disproved through the data gathered in the existing trolley network. The issue of corridor sizes and integration had already been established as a prime objective of the project. Finally end to end loading and unloading is currently handled manually in the existing system with very few problems. This should be no different when transitioning from manual trolleys to AMRs.

\subsection{Current applicable safety standards}

All buildings in the UK have been required to meet BSs since 1984 when the first regulations were brought into effect. These were typically to ensure the structure and safety of occupants, protecting the legal rights of those harmed due to negligence. There are sixteen instances from BS 9999 [12] where a mobile robot traveling through a densely occupied building could be in violation of these standards in the event of an accident or loss of life. These are listed in Table 1.

Table 1. A selection of BS 9999 (Handbook - Effective Fire Safety in the Design, Management and Use of Buildings) safety standards that may require special consideration when using AMR's in common areas.

\begin{tabular}{ll}
\hline \multicolumn{1}{c}{ Building Standard 9999 sections that overlap with AMR use } \\
\hline 8.3.1 Implementing fire risk management strategy: & 10.3 .1 Housekeeping \\
General & 10.3 .3 Security \\
8.3.3 Resources and authority & 10.4 .1 .5 Other factors \\
8.3.4 Fire safety training & 10.4.1.5 Fire doors and escape routes \\
8.3.7 Communication & 11 Principles of means of escape \\
8.3.8 Emergency planning & 14.2 Acceptable means of escape \\
9 Fire safety documentation & 15.4 Artificial and emergency escape lighting \\
10.2.1 Management input: General & 16.6.2 Corridors and escape routes \\
10.2.2 Designing for the management of means of &
\end{tabular}

These instances have also been further broken down into 4 main categories: 
- Preplanning: where health and safety officers continually check that the infrastructure is in place and in working order prior to an emergency.

- Evacuation procedure: All staff and visitors should be made aware of this procedure and it should be updated continually over time.

- Resources: referring specifically to alarms, fire hydrants, smoke ventilation and any other equipment used during the emergency.

- Communication: Allowing both internal communication between trained/accredited safety officers and emergency services personnel.

Within these categories some overlap occurs the regulation and what they are pertaining to. This can be seen in the Venn diagram shown in Figure 1.

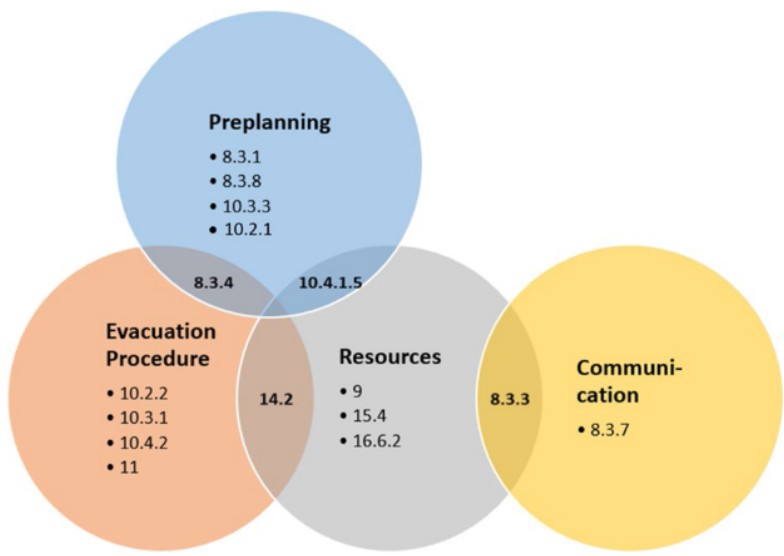

Figure 1. Venn diagram of categorised building regulations.

In regards to the HSE which is Britain's national regulator for workplace health, there is one primary document related to driverless vehicles [14]. In the report article 59 states three key requirements of driverless vehicles. The first is that "...make sure vehicles are not able to trap pedestrians.". This specifically is to be considered within a building by not creating pinch points in corridors that could trap workers or by not blocking doorways.

"... make sure fixtures along the route do not create trapping hazards." Buildings corridor width are fairly inconsistent. This can make it difficult for staff to pass around a robot safely and should be considered in the analysis of what makes robot travel safe.

"... vehicles should be fitted with safeguards to keep the risk of injury low if they hit someone." This is typically completed by the manufacturer by providing a casing around any metal framework and covering jagged electronics that would otherwise be exposed.

\section{Conclusion and Further Work}

The question of justification remains partially unanswered for BAE Systems. The initial phases of this study show that AMRs can provide a substantial number of hours back to the working schedules of many skilled engineers. As they do not employ dedicated trolley pullers, the use of AMRs would also not endanger the jobs of any individual. It can also be seen that staff are fairly trusting of the technology with their concerns being 
addressable. However, the issue of BS, HSE and ISO standards remain a concern, especially as the academic and commercial solutions for improved global navigation and robotic awareness can involve the installation of costly sensor network or cameras that violate the sites security protocols. It has been suggested that this final hurdle can be overcome by using existing sensor networks from within the building's internet of things (IoT) to feed the robot with real time data on anonymous occupant locations. Once trialled and tested against the standards raised in this paper BAE Systems can have full justification for the use of robots in this environment.

\section{Acknowledgements}

This project was jointly funded by EU Interreg V France Channel England Programme (CoRoT, Ref: 99) and BAE Systems. A special recognition to the engineers and managers from BAE Systems and the University of Greenwich academics who provided advice and guidance throughout the project.

\section{References}

[1] Giuseppe Fragapane, René de Koster, Fabio Sgarbossa, Jan Ola Strandhagen, Planning and control of autonomous mobile robots for intralogistics: Literature review and research agenda, European Journal of Operational Research, Volume 294, Issue 2, (2021) Pages 405-426, ISSN 0377-2217

[2] N. J. Hetherington, E. A. C. a. H. F. M. V. d. L. "Hey Robot, Which Way Are You Going? Nonverbal Motion Legibility Cues for Human-Robot Spatial Interaction,". IEEE Robotics and Automation Letters,, vol. 6,(no. 3,), (2021). pp. 5010-5015.

[3] C. Lichtenthäler, T. L. a. A. K. "Influence of legibility on perceived safety in a virtual human-robot path crossing task,". s.1., IEEE RO-MAN: The 21st IEEE International Symposium on Robot and Human Interactive Communication, (2012)

[4] De Silva, V. R. J. a. K. A., . Robust Fusion of LiDAR and Wide-Angle Camera Data for Autonomous Mobile Robots.. Sensors, (2018)

[5] Pourrahimian, P., A new memetic algorithm for mitigating tandem automated guided vehicle system partitioning problem. Journal of Industrial Engineering International. (2017)

[6] Draganjac, I. P. T. M. D. K. Z. a. O. J., Highly-scalable traffic management of autonomous industrial transportation systems. Robotics and Computer-Integrated Manufacturing. (2020)

[7] Dias, L. d. O. S. R. d. S. E. P. F. A. a. B. R., . Application of the Fuzzy Logic for the Development of Automnomous Robot with Obstacles Deviation.. International Journal of Control, Automation and Systems, (2018).

[8] Sahnoun, M. X. Y. A. F. a. B. D., Optimization of Transportation Collaborative Robots Fleet Size in Flexible Manufacturing Systems. s.1., 8th International Conference on Modeling Simulation and Applied Optimization (ICMSAO) (2019).

[9] Martin, Lancelot, González-Romo, Mario, Sahnoun, M'hammed, Bettayeb, Belgacem, He, Naihui and Gao, James, Effect of Human-Robot Interaction on the Fleet Size of AIV Transporters in FMS. In: 2021 1st International Conference On Cyber Management And Engineering (CyMaEn). IEEE. (In Press) (2021)

[10] Fosch Villaronga, E. a. G. A. Robots, standards and the law: Rivalries between private standards and public policymaking for robot governance. Computer Law \& Security Review, 35(2), , (2019) pp. pp.129144.

[11] House of Lords Select Committee on Artificial Intelligence, AI in the UK: ready, willing and able, Published by the Authority of the House of Lords, (2018)

[12] British Standards, BS 9999: Fire safety in the design, management and use of buildings. Code of practice. s.1.:BSI. (2017)

[13] Health and Safety Executive . RR906: Collision and injury criteria when working with collaborative robots, s.1.: Health and Safety Executive. (2012)

[14] Health and Safety Executive. A guide to workplace transport safety, s.1.: Health and Safety Executive. (2014)

[15] ISO/TS 15066, Robots and robotic devices, s.1.: International Organization for Standardization. (2016) 